\title{
LA RESPONSABILIDAD PLENA DE PERJUICIOS Y EL SISTEMA GENERAL DE RIESGOS LABORALES. PRECEDENTE JUDICIAL DE LA CORTE SUPREMA DE JUSTICIA 2019
}

\author{
FULL LIABILITY FOR DAMAGES AND WORKERS' \\ COMPENSATION SYSTEM. \\ JUDICIAL RECORD OF THE SUPREME COURT OF \\ JUSTICE 2019
}

GERMÁN ERNESTO PONCE BRAVO*

Fecha de recepción: 30 de agosto de 2018

Fecha de aceptación: 4 de septiembre de 2019

Disponible en línea: 15 de septiembre 2019

Para citar este artículo/To cite this article

Ponce Bravo, Germán Ernesto, La responsabilidad plena de perjuicios y el sistema general de riesgos laborales. Precedente judicial de la Corte Suprema de Justicia 2019, 50 Rev.Ibero-Latinoam.Seguros, 177-205 (2019). https://doi. org/10.11144/Javeriana.ris50.rpps

doi.org/10.11144/Javeriana.ris50.rpps

* Abogado de la Universidad Externado de Colombia, Magíster en Derecho Administrativo y en Dirección y Gestión de los Sistemas de Seguridad Social, de las Universidades del Rosario y Alcalá (España). Actualmente se desempeña como director de la Cámara Técnica de Riesgos Laborales de Fasecolda, fue subdirector de Prestaciones Económicas del Fondo de Pensiones de Bogotá, así como gerente nacional de doctrina en la Vicepresidencia Jurídica de Colpensiones. Es docente de postgrado en las Universidades Externado y La Sabana. Contacto: gponce@fasecolda.com 


\section{RESUMEN}

El elemento que diferencia el seguro de riesgos laborales del resto de seguros es la intervención del riesgo. A mayor intervención del riesgo, menor la siniestralidad esperada. Una de las herramientas de intervención del riesgo es el sistema de seguridad y salud en el trabajo, que además es uno los principales criterios adoptados por la Corte Suprema de Justicia, en el año 2019, para deducir la culpa del empleador no solo por el incumplimiento de las normas de seguridad y salud en el trabajo. La reflexión es que la distancia que media entre la responsabilidad objetiva y subjetiva es cada vez más difusa.

Palabras clave: sistema general de riesgos laborales; seguro; responsabilidad objetiva; responsabilidad plena de perjuicios; Artículo 216 del C.S.T; seguridad y salud en el trabajo. 


\begin{abstract}
The element that differentiates occupational risk insurance from other insurance is risk intervention. The greater the risk intervention, the lower the expected sinistrality. One of the risk intervention tools is the occupational health and safety system, which is also one of the main criteria adopted by the Supreme Court of Justice, in 2019, to deduce the employer's guilt not only for non-compliance with the occupational health and safety regulations. The conclusion is that the distance between objective and subjective responsibility is increasingly diffuse.
\end{abstract}

Keywords: workers compensation; insurance; security and health at work, objective responsability

\title{
SUMARIO
}

SUMARIO: INTRODUCCIÓN - 1. LA OBLIGACIÓN GENERAL DE PROTECCIÓN Y SEGURIDAD PARA CON LOS TRABAJADORES2. LAS REGLAS DE LA CORTE SUPREMA DE JUSTICIA PARA DETERMINAR LA RESPONSABILIDAD PLENA DEL EMPLEADOR. PRECEDENTE JUDICIAL 2019 - CONCLUSIONES - REFERENCIAS BIBLIOGRÁFICAS 



\section{INTRODUCCIÓN}

El objetivo de este artículo es identificar los principales criterios adoptados por la Corte Suprema de Justicia, en el año 2019, para deducir la culpa del empleador no solo por el incumplimiento de las normas de seguridad y salud en el trabajo, sino también por la falta de cautela necesaria para evitar la materialización de siniestros en el trabajo.

En este orden de ideas, el riesgo creado por el empleador a la salud de los trabajadores le obliga a implementar un sistema que sea consecuente con el objetivo propio de la empresa: generar productividad, pero también que permita el control de los riesgos laborales a los que expone a su personal de trabajo. El desarrollo de la actividad económica de la empresa conlleva la adopción de medidas necesarias para prevenir la ocurrencia de una contingencia de origen laboral, obligación legal que impone la ley 1562 de 2012, el Decreto 1072 de 2015ํㅡㄹ, específicamente, la resolución 312 de 2019 del Ministerio del Trabajo.

El logro de este cometido requiere de una actuación coordinada de cada uno de los actores del sistema, obligación que comienza en la dirección general de la empresa, pasando por la cadena de mando y operaciones de los trabajadores, el COPASST y las Administradoras de Riesgos Laborales (ARL).

Es importante señalar que las administradoras de riesgos laborales tienen el deber de realizar la socialización del sistema a través de asesorías técnicas, capacitaciones y acompañamiento en la implementación, sin que dicha labor implique asunción de las actividades que le corresponden al empleador y, que no pueden ser trasladadas a las ARL para cubrir necesidades internas de la empresa o entidad².

En este orden de ideas, conocer la evolución del seguro de riesgos laborales es importante para contextualizar el alcance de las decisiones

$1 \quad$ Ver Capítulo 6 del Título 4 de la Parte 2 del Libro 2.

2 Ley 1562 de 2012, artículo 11 parágrafo 1: "Las administradoras de riesgos laborales no pueden desplazar el recurso humano ni financiar las actividades que por ley le corresponden al empleador, y deben otorgar todos los servicios de promoción y prevención sin ninguna discriminación, bajo el principio de la solidaridad, sin tener en cuenta el monto de la cotización o el número de trabajadores afiliados." 
judiciales adoptadas por la Corte Suprema de Justicia en el primer semestre de 2019.

El sistema de seguridad social en el ámbito de los riesgos laborales se origina en la necesidad de proteger al trabajador que se encuentra expuesto a contingencias en su ambiente laboral que pueden afectar su salud. De esta manera, la responsabilidad por la seguridad de los trabajadores se atribuye al empleador, bajo el principio de que toda actividad productiva o de servicios comprende riesgos (Conte-Grand, 1999). Esto explica por qué la esencia de la protección social del riesgo laboral es la prevención de los riesgos, antes que la reparación de los daños; por esta razón, en la última década el esfuerzo regulatorio de este sistema se ha concentrado en transformar los programas de salud ocupacional en un sistema de gestión integrado a la operación diaria (Velásquez, 2016).

La evolución normativa del Sistema General de Riesgos Laborales (SGRL) en Colombia ha sido sustancial y, actualmente, la más importante se concreta en el modelo de trasferencia del riesgo, en el marco de libre elección y competencia, previsto en el Decreto 1295 de 1994, la Ley 776 de 2002, la Ley 1562 de 2012 y sus decretos reglamentarios. El consenso doctrinal sobre la evolución legal del modelo de protección de los riesgos laborales permite identificar cinco etapas o fases (Cortés, 2015). Cada una de estas conllevó un cambio importante frente a la anterior, pues las necesidades de protección del sistema corren de manera simultánea con la evolución del sistema productivo y las relaciones laborales (Figura 1)

Figura 1. Evolución del sistema general de riesgos laborales.

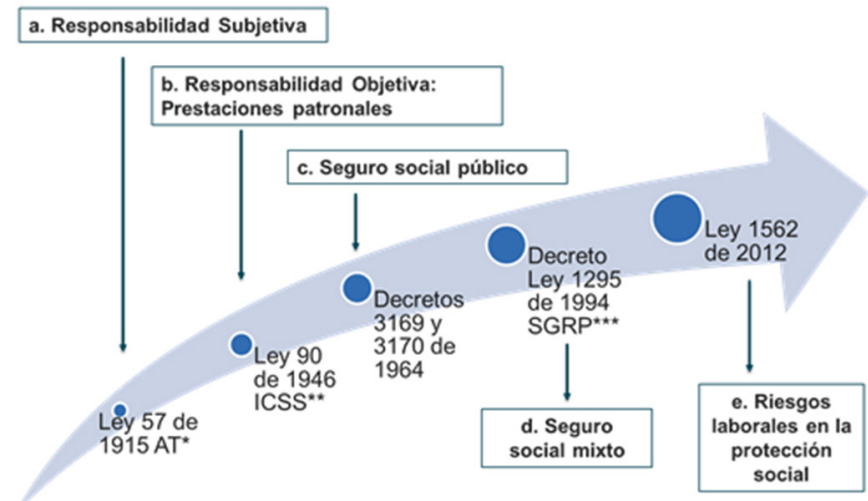

Fuente: Cortés (2015). 
En síntesis, existen dos tipos de teorías sobre el fundamento jurídico de la responsabilidad patronal derivada de los accidentes de trabajo y enfermedades laborales, clasificadas como subjetivas y objetivas. Este tránsito se evidencia en la evolución normativa colombiana de riesgos laborales (Arenas, 2011). Las teorías subjetivas señalaban que para determinar la responsabilidad del empleador solo debe considerarse la culpa o la vinculación de las personas en un accidente de trabajo o una enfermedad laboral. De otro lado, las teorías objetivas se fundamentan en que toda actividad material implica para quien la ejecuta o contribuye a ejecutarla un riesgo determinado, lo que genera presunción de responsabilidad a cargo de quien está al frente de dicha actividad o se beneficia de ella (Rodríguez M., 2016).

En este orden de ideas, la Ley 57 de 1915 consagró el reconocimiento de: prestaciones médico-asistenciales por accidentes de trabajo y enfermedad profesional; responsabilidad del empleador; tipos de incapacidades; indemnizaciones a cargo del empleador, cuando el trabajador quedara en su integridad física a causa de una contingencia originada en el trabajo (Gómez C., 2015).

Esta consagración normativa rompió con la antigua tradición civilista del Código Civil napoleónico de 1804, según la cual, por ejemplo, el tratamiento "laboral" de los "criados domésticos" era bajo la figura del arrendamiento de servicios. Este principio “... será modificado por el concepto de contrato de trabajo, sobre el cual se basarán las futuras leyes laborales. La legislación laboral del continente [...] aportó nuevas instituciones jurídicas, como las relacionadas con las indemnizaciones por accidentes de trabajo; en el caso de Colombia, las Leyes 57 de 1915 y 133 de 1931 modificaron el criterio tradicional sobre la Culpa y la Responsabilidad Civil que albergaba el Código Civil." (Avella, 2012)

En este orden de ideas, “... los antiguos rudimentos de legislación laboral venían incorporados en la civil y en la comercial, de acuerdo con la tradición jurídica internacional. En América Latina la opción acogida por varios países fue la de la codificación de las leyes laborales, tras procesos jurídicos y políticos prolongados. Colombia siguió dicho camino; lo emprendió a mediados de los veinte, y después de algunos intentos malogrados o parciales pudo expedir una Ley General 
del Trabajo en 1945, y finalmente el Código del Trabajo en 1950. Este largo devenir estuvo precedido por un periodo de producción de leyes sociales aisladas, concentrado entre 1915 y 1930, al igual que en el resto de América Latina". (Avella, 2012)

El seguro público fue la respuesta a la necesaria evolución conceptual del riesgo laboral que se estaba dando mundialmente. De esta manera, a partir de normas como la Ley 90 de 1946 se unificó la legislación de seguridad social, se pretendió ampliar la cobertura y, especialmente, se aplicó la noción de seguro obligatorio por razón del trabajo subordinado, lo que trajo como consecuencia migrar hacia la responsabilidad objetiva del sistema (Cortés G., 2015).

Es importante resaltar que durante esta época la consolidación normativa fue considerable. Por esta razón, entre sus normas más representativas, la Ley 6 (Congreso de Colombia, 1945) fue la punta de lanza de la seguridad social previsional, pues creó la Caja Nacional de Previsión Social para los trabajadores de la nación y consolidó el contrato de trabajo y la ordenación de las relaciones individuales y colectivas.

En esa misma línea, como se mencionó anteriormente, tan solo con un año de diferencia se expidió la Ley 90 (Congreso de Colombia, 1946), cuyo hito más importante fue la creación del Instituto Colombiano de Seguros Sociales (ICSS). Una de las particularidades más importantes de esa entidad consistía en que se daba sus propios reglamentos, que posteriormente se convertían en decretos. De manera que por esta vía se avanzó en la protección de los derechos del trabajador asegurado frente a un accidente de trabajo o enfermedad profesional.

Entre 1950 y 1969 se expidieron importantes normas que dieron el fundamento de lo que hoy es el seguro de riesgos laborales, entre otras, las más importantes son (García, 2018) : Los Decretos 2663 y 3743 (Presidencia de la República, 1950a/b) por los cuales se precisaron, bajo el concepto de responsabilidad objetiva, los conceptos de accidente de trabajo, enfermedad profesional y sus prestaciones. Es necesario llamar la atención respecto del hecho de que el Código Sustantivo del Trabajo no estableció pensiones de invalidez ni de sobrevivencia y que su novedad y avance dentro de la estructuración del SGRL colombiano consistió en 
que su artículo 209 estableció la tabla de valuación de incapacidades resultantes de accidente de trabajo para determinar la suma a pagar por algunas de las prestaciones económicas establecidas y la adopción de una tabla de enfermedades profesionales en su artículo 201.

El Decreto 3169 (Presidencia de la República, 1964a) se convirtió en el estatuto de seguridad social laboral, pues reguló en detalle la afiliación al sistema (que por la época se denominó "inscripción") y también se estrenó con la clasificación de empresas y de los aportes obligatorios que financiarían el seguro. De otro lado, el Decreto 3170 (Presidencia de la República, 1964b) abordó nuevamente las definiciones de accidentes de trabajo (AT) y enfermedades profesionales (EP) y concretó cuales eras las excepciones que no se consideraban AT. El decreto solo se aplicó a los trabajadores nacionales o extranjeros que, en virtud de un contrato de trabajo, prestaran servicios a empleadores del sector privado de la economía. Estos fueron derogados por el Acuerdo 044 de 1989, aprobado por el Decreto 3063 de ese mismo año, que sería la herramienta normativa definitiva, antes de la expedición del Decreto 1295 de 1994.

Señalamos desde el inicio de esta sección que el elemento central del sistema es la prevención del riesgo y la promoción de la salud laboral. Adoptar el mecanismo de seguro obligatorio fue una decisión consistente y con máximos beneficios para el país, porque generó la conciencia de reglamentar la prevención en materia de riesgos laborales bajo el Acuerdo 241 de 1967 (ICSS, 1967).

Los Decretos 3135 y 1848 (Presidencia de la República, 1968/1969) definieron las normas aplicables para los riesgos laborales de los servidores públicos y la Ley 12 (Congreso de Colombia, 1977) otorgó al presidente la potestad de modificar la estructura, régimen y organización de los Seguros Sociales Obligatorios. El documento de la propuesta de reestructuración presentaba un panorama del porqué de las transformaciones planteadas, que es una buena descripción de la situación de la seguridad social en el país hasta ese momento. Ese informe dice:

"La Seguridad Social ha adolecido en su proceso histórico de definiciones sobre lo que ella misma es, y sobre sus objetivos. Por esta razón el sector responsable de la seguridad social se encuentra disperso en 
múltiples entidades, adscritas a distintos ministerios, con ausencia absoluta de integración y coordinación. Lo antes dicho, aunado a la falta de un sistema de seguridad social, ha impedido que se tengan objetivos claros sobre sus responsabilidades frente a la comunidad, que se establezcan politicas para una cobertura integral de sus contingencias, que se asignen los medios necesarios para alcanzar un grado aceptable de bienestar social. Corrobora lo anterior el desconocimiento de los programas de seguridad social en los planes de desarrollo que ha tenido el país."

En el marco descrito en el párrafo anterior, se emitió la Ley 9 (Congreso de Colombia, 1979), como un esfuerzo del Estado por estructurar un Código Sanitario. Esa ley comprendió medidas sanitarias en múltiples temas, dentro de los cuales dedicó una la salud ocupacional. Junto con esta ley, son piedras angulares ligadas a los riesgos profesionales: la Resolución 2400 de 1979, que estableció disposiciones sobre los establecimientos de trabajo, su higiene y seguridad; el Decreto 614 de 1984, que sentó las bases para la organización y administración de la salud ocupacional en el país; la Resolución 2013 de 1986, que reglamentó la organización y funcionamiento de los comités de medicina, higiene y seguridad industrial en los lugares de trabajo; y, posteriormente, la resolución 1016 de 1989, que reglamentó la organización, funcionamiento y forma de los programas de salud ocupacional que debían desarrollar los patronos o empleadores en el país.

La evolución del sistema determinó la necesidad de ampliar su alcance y mejorar la prestación del servicio. Así las cosas, el Sistema General de Riesgos Profesionales (SGRP) se consagró -en su versión ligada a la competencia y fuera del estricto monopolio estatal- en el Libro III de la Ley 100 (Congreso de la República, 1993), artículos 249 a 256. Estos fueron reglamentados por el Decreto Ley 1295 (Presidencia de la República, 1994), por el cual se transformó la organización y administración del SGRP y determinó su estructura actual. El decreto Ley 1295 definió el accidente de trabajo de la siguiente manera:

Es accidente de trabajo suceso repentino que sobrevenga por causa o con ocasión del trabajo, y que produzca en el trabajador una lesión orgánica, una perturbación funcional, una invalidez o la muerte. Es también 
accidente de trabajo aquel que se produce durante la ejecución de órdenes del empleador, o durante la ejecución de una labor bajo su autoridad, aún fuera del lugar y horas de trabajo. Igualmente se considera accidente de trabajo el que se produzca durante el traslado de los trabajadores desde su residencia a los lugares de trabajo o viceversa, cuando el transporte lo suministre el empleador.

En esta etapa se cerró la discusión o evolución sobre la responsabilidad patronal por los riesgos laborales, concluyó la viabilidad de la responsabilidad objetiva y se concretó el alcance conceptual del accidente de trabajo. De esta manera, el tránsito normativo del sistema que se inició con el seguro público a través del Acuerdo 155 de 1963 del ISS (aprobado por el Decreto 3170 de 1964), el Decreto 3135 de 1968, el Decreto 1848 de 1969, hasta llegar a la expedición de la vigente Ley 100 de 1993, que creó el Sistema General de Riesgos Profesionales -asumiendo las contingencias generadas por accidentes de trabajo y enfermedad profesional-, reglamentado en 1994 por los Decretos 1295, 1771, 1772, el primero de los cuales, por ser declarado parcialmente inexequible por la Corte Constitucional en Sentencia C-452 de 12 junio de 2002, dio lugar a la Ley 776 (Congreso de Colombia, 2002), "por la cual se dictan normas sobre la organización, administración y prestaciones del Sistema General de Riesgos Profesionales", recogiendo en términos similares los apartes declarados inexequibles del Decreto 1295 de 1994 por vicios de forma. En síntesis, su estructura consagró los siguientes tópicos:

1. Define que todo afiliado que padezca un accidente de trabajo o enfermedad laboral (profesional, en ese entonces) tiene derecho a las prestaciones asistenciales y económicas previstas en el sistema.

2. Otorga la competencia al gobierno nacional para establecer un régimen de reservas, cuyo objetivo es contar con el capital suficiente para cubrir los siniestros de salud o económicos de los beneficiarios.

3. Contempla la viabilidad de suspensión del servicio cuando el afiliado o pensionado no se someta a exámenes controles o prescripciones ordenadas por la administradora de riesgos. 
4. Fija que los traslados entre administradoras sean: para Positiva, antes ISS, después de dos años, contados desde la última afiliación o traslado. Para las demás, un año. Los efectos de traslado serán a partir del primer día del mes siguiente a que se produjo el traslado.

El desarrollo jurisprudencial que desplegó la Corte Constitucional en materia de riesgos laborales fue considerable y se profirieron diversas sentencias, por las cuales se ordenó la regulación legal del sistema. Una de las más importantes fue la C-858 de 2006, en la que evidenció un exceso en el uso de las facultades otorgadas al poder ejecutivo con la expedición del Decreto Ley 1295 de 1994. En las demandas de inconstitucionalidad contra los artículos 9, 10 y 13 parcial de este decreto, el argumento fue contundente:

En atención a la importancia que para la estabilidad del Sistema General de Riesgos Profesionales revisten las normas impugnadas y con el fin de mitigar los efectos inmediatos de una decisión de inexequibilidad, la Corte atenderá la solicitud del Procurador General de la Nación y de algunos de los intervinientes en el sentido de diferir los efectos de esta decisión por el término de ocho (8) meses, hasta el veinte (20) de junio de 2007, a fin de que el Congreso expida una Ley que defina los aspectos declarados inexequibles en el presente proceso .

En esa ocasión, la Corte difirió los efectos de la sentencia hasta el final de la legislatura de 2007, con el objetivo de que el Congreso expidiera una ley que ocupara los vacíos dejados por la inexequibilidad declarada, evitando así un caos en el SGRP colombiano. Este proceso duró más de cinco años sin ningún avance. Por el contrario, nuevamente, la Corte intervino declarando la inexequibilidad del artículo que establecía el ingreso base para liquidar las prestaciones económicas del sistema:

Una vez confrontadas la disposición acusada y la norma legal habilitante, teniendo además en cuenta el precedente jurisprudencial sentado en la sentencia C-452 de 2002, M.P. Jaime Araujo Rentería, comparte la Corte el concepto del Ministerio Público, en el sentido de que el Gobierno Nacional, mediante la expedición del artículo 20 del decreto ley 1295 de 1994, se excedió en el ejercicio de las facultades extraordinarias, ya que la delegación legislativa se concedió, de manera precisa y clara, 
únicamente para organizar la administración del Sistema General de Riesgos Profesionales y no para regular un aspecto diferente como el de carácter sustantivo referente al ingreso base que servirá para liquidar las prestaciones económicas causadas por efectos de un accidente de trabajo o una enfermedad profesional, es decir, el ingreso base que se empleará para efectos de reconocer y cancelar el subsidio por incapacidad temporal, la indemnización por incapacidad permanente parcial, la pensión de invalidez, la pensión de sobrevivientes y el auxilio funerario.

Como se ha indicado, la habilitación legislativa contenida en el numeral 11 del artículo 139 de la Ley 100 de 1993, tenía como propósito específico facultar al Gobierno para dictar normas relacionadas exclusivamente con la organización de la administración del Sistema General de Riesgos Profesionales, siendo por tanto el ingreso base de liquidación de las prestaciones sociales un tema ajeno e independiente a aquél para el cual había sido habilitado el Gobierno Nacional.

La consecuencia de esta decisión fue la de generar un vacío normativo imperioso en materia de riesgos laborales. La reacción fue implementar el Instrumento Andino de Seguridad y Salud en el Trabajo, adoptado por la Comunidad Andina de Naciones (CAN), mediante la Decisión 584 de 2004 (Consejo de Ministros de Relaciones Exteriores, 2004).

En la Sentencia C-1155 de 2008, en su parte considerativa, se dijo que, como se declaraba la inexequibilidad del artículo 11 del Decreto Ley 1295 de 1994, debía revivir el ordenamiento anterior con relación al concepto de enfermedad profesional, es decir, el artículo 200 del Código Sustantivo del Trabajo (CST). Comparando la definición del inexequible artículo 11 del Decreto Ley 1295 de 1994 con la del artículo 200 del CST, se observa que en esta última no se contempló lo relativo a la relación de causalidad con los factores de riesgo ocupacionales en los casos en que una patología no figure en la tabla de enfermedades profesionales (García, 2018). Por este motivo, el gobierno nacional derogó el Decreto 778 (Presidencia de la Repúbica, 1987) con la expedición del Decreto 2566 (Presidencia de la República, 2009) por el cual se adoptó la nueva tabla de enfermedades profesionales, que son las mismas contempladas en el Decreto 1832 de 1994. Este adoptó el criterio causal para determinar el origen de la contingencia; de esta manera, para que se reconozca una 
enfermedad profesional, actualmente laboral, no importaba si ella no se encontraba relacionada en listado de 42 enfermedades que contenía el decreto, siempre que se demostrara la relación de causalidad con los factores de riesgo ocupacional. La relación de causalidad se determina con la identificación de dos elementos: a) la presencia de un factor de riesgo causal ocupacional en el sitio de trabajo en el cual estuvo expuesto el trabajador y b) la presencia de una enfermedad diagnosticada médicamente relacionada causalmente con ese factor de riesgo.

Finalmente, se expidió la Ley 1562 (Congreso de Colombia, 2012) por medio de la cual se modificó el SGRP que reemplazó el nombre de Sistema General de Riesgos Profesionales por el de Sistema General de Riesgos Laborales (SGRL). La doctrina nacional señala que los cambios adoptados por esta ley marcaron un hito en la historia del sistema, que ahora le definen características específicas, entre las más importantes están (Cortés G., 2015):

1. Se trata de un sistema menos judicializado que los otros dos subsistemas de salud y pensiones.

2. Se soporta en un esquema controlado de reservas que aseguran el cubrimiento de las prestaciones que se causen. En ese sentido, el modelo financiero, contable, administrativo y técnico permiten que las ARL cuenten con la solvencia necesaria para cubrir las atenciones médicas y económicas.

3. Es un sistema que tiende de manera progresiva a la inclusión del sector independiente.

El avance de esta norma frente al Decreto 1295 se evidenció en varios aspectos: a) en una definición más amplia de accidente laboral, pues la perturbación puede ser funcional o psiquiátrica; b) en que el accidente es de origen laboral si se produce durante el traslado de los trabajadores o contratistas desde su residencia a los lugares de trabajo o viceversa, cuando el transporte lo suministre el empleador; c) en que el accidente también ocurre durante el ejercicio de la función sindical, aunque el trabajador se encuentre en permiso sindical, siempre que se dé en cumplimiento de dicha función; y d) en que se extiende la consideración 
de accidente de trabajo a los trabajadores en misión cuando actúen por cuenta o en representación de la empresa usuaria, cuando ocurre por la realización de actividades recreativas, deportivas o culturales. Con la expedición de la Ley 1562 de 2012, desapareció la necesidad de acudir a la definición de la CAN.

En cuanto a la definición de enfermedad laboral, se ordena la actualización permanente de la tabla de enfermedades laborales por un término no menor a tres años. Eso explica la aparición del Decreto 1477 (Presidencia de la República, 2014). En esta oportunidad la nueva tabla de enfermedades laborales es más extensa y detallada que la anterior - establecida por el Decreto 2566 de 2009- y fija los agentes de riesgo para facilitar la prevención de enfermedades en las actividades laborales y los grupos de enfermedades para determinar el diagnóstico médico en los trabajadores afectados.

La Ley 1443 (Presidencia de la República, 2014) dicta disposiciones para la implementación del Sistema de Gestión de la Seguridad y Salud en el Trabajo (SGSST), cuyo término de aplicación fue ampliado, primero mediante el Decreto 171 de 2016 hasta el 31 de enero de 2017 y posteriormente por el Decreto 52 de 2017. Así las cosas, entre junio de 2017 y abril de 2019 se habrá implementado la aplicación del Sistema de Gestión, de acuerdo con las fechas que se especifican en la Figura 2, así:

Figura 2. Fases de la aplicación del SGSST

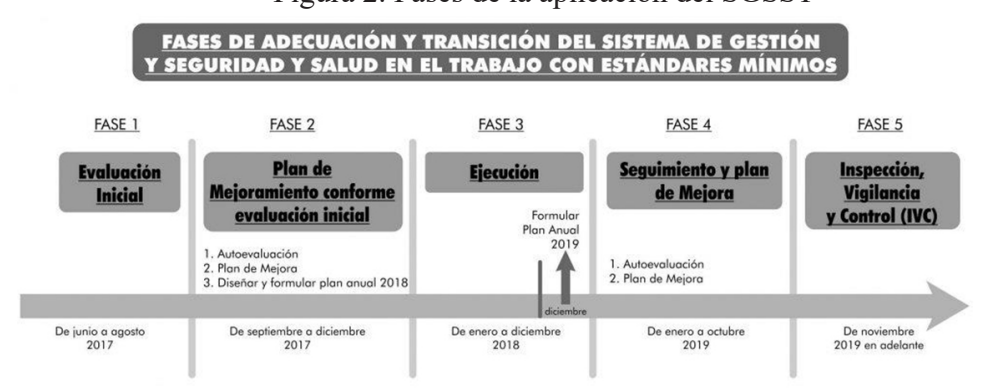

La ampliación del plazo inicialmente previsto por el Decreto $171 \mathrm{de}$ 2016 fue de seis meses. El objetivo es que las empresas del país inicien la implementación del Sistema de Gestión a partir del 1 de junio de 2017 
y se logre la implementación del sistema por parte del Estado, como empleador y mayor contratante del país, y de las empresas del sector privado, sin considerar su tamaño y ni el número de trabajadores.

El elemento conceptual básico de esta normativa radica en la posibilidad de identificar los peligros, evaluar y valorar los riesgos y establecer los respectivos controles, mediante la mejora continua del Sistema en las empresas para controlar las contingencias que puedan afectar la seguridad y la salud en el trabajo. En este orden de ideas, a partir del 1 de junio de 2017, la implementación del Sistema de Gestión se debe ejecutar de manera progresiva y sistemática en cinco fases: evaluación inicial, plan de mejoramiento, ejecución, seguimiento y plan de mejora, más la fase de inspección, vigilancia y control, de acuerdo con el cronograma que establece el Ministerio del Trabajo, en la Resolución 312 de 2019 sobre estándares mínimos del Sistema de Gestión de la Seguridad y Salud en el Trabajo, para lo cual las empresas deben contar con los soportes, antecedentes y pruebas de ejecución. Estos son sus lineamentos básicos:

Es un proceso lógico y por etapas, basado en la mejora continua, con el objetivo de anticipar, reconocer, evaluar y controlar los riesgos que puedan afectar la seguridad y salud en el trabajo.

- El primer objetivo de la implementación del sistema es prevenir las lesiones y enfermedades causadas por las condiciones de trabajo a las cuales están expuestos los trabajadores y contratistas, reduciendo al mínimo los accidentes de trabajo y enfermedades laborales.

- La implementación del SGSST debe ser liderada por el empleador con la participación de los trabajadores; de otra manera no se lograría la aplicación de las medidas adecuadas de prevención y control eficaz de los peligros y riesgos en el lugar de trabajo.

- El deber de las ARL es, esencialmente, el de asesorar y capacitar en las diferentes fases de implementación del Sistema de Gestión a los afiliados obligados a adelantar este proceso. 
Ahora bien, el hito más importante en términos normativos, económicos y de eficacia del Sistema General de Riesgos Laborales en Colombia se encuentra en la concepción de este como un modelo de aseguramiento, lo cual trae varios beneficios:

1. El seguro de riesgos laborales implica su transferencia desde el empleador a la ARL, es decir, el empresario no tiene que pagar emergencias de salud o pensiones por invalidez o muerte de sus empleados.

2. Fortalece la necesidad de generalizar una cultura preventiva a través de una gestión participativa, dinámica, compartida y abierta del riesgo dentro de la organización laboral.

3. Los empleadores encuentran hoy un sistema de riesgos laborales activo y competitivo, que potencia los principios de eficacia, integralidad, universalidad y solidaridad de la protección social ${ }^{3}$ (Cañón, 2017).

La regla general de responsabilidad por causación de un daño consiste en que quien genera el daño debe repararlo; sin embargo, en materia de riesgos laborales este principio no es aplicable, pues requiere probar que el empleador es el agente generador del riesgo. En consecuencia, se recurrió a la noción de responsabilidad objetiva del empleador por el riesgo creado o riesgo de exposición, de manera que dicha "responsabilidad no se funda en que el responsable haya obrado injustamente, sino en que está obligado a soportar en virtud de la ley el riesgo de producir un daño para otro unido a una actividad autorizada" (Larenz, 1958)

3 De acuerdo con Leonardo Cañón (2017), la manifestación del principio de solidaridad en el Sistema de Riesgos Laborales se encuentra, entre otros, a) en los aportes que hacen los empleadores que desarrollan actividades económicas y de diversas clases y grado de riesgo, para dar protección a los afiliados y sus beneficiarios contra los riesgos laborales, y b) en la parte relacionada con las medidas de prevención y control de los riesgos laborales y promoción de actividades de autocuidado, dentro del Sistema de Gestión en Salud y Seguridad en el Trabajo (SGSST), financiadas con parte de la prima del seguro para beneficio de todos los trabajadores y contratistas, así sean aquellos que no se accidenten ni adquieran enfermedad laboral alguna.

4 Este concepto fue especialmente desarrollado por la ley francesa de accidentes de trabajo de 1896 y por la doctrina francesa en Les accidents du travail et la responsabilitè civile, de Raymond Saleilles (1897) y De la responsabilitè du fait des choses, de Louise Josserand (1898). 
El empleador no debe responder de manera individual por una labor que representa un bien colectivo, como el trabajo. Por esta razón, cuando se realiza el siniestro laboral se recurre a una indemnización del daño tarifada, es decir, que se trata de una indemnización cuyo valor esta previamente señalado en la ley (Valdivieso y Lombana, 2014)

Este concepto responde a la dispersión del riesgo ocupacional entre todos los empleadores y la razón es que los accidentes de trabajo y las enfermedades laborales son producto de una actividad común de todos los empleadores, por lo que su reparación debe ser responsabilidad de todos ellos. De esta manera, los empleadores se hacen responsables colectivamente de los daños producidos, para lo cual deben asegurarse previamente frente a un infortunio laboral (Puyana, 2013). Por lo tanto, el riesgo laboral es una actividad aseguradora (Valdivieso y Lombana, 2014) que implica ${ }^{6}$ :

1. Interés asegurable del empleador por el eventual daño que sufra el trabajador por causa o con ocasión al trabajo. Este interés se materializa en la indemnización que reconoce y paga la Administradora de Riesgos Laborales al trabajador.

2. Una prima, entendida como el valor o precio que cobra la ARL por asumir los riesgos que le traslada el empleador asegurado, es decir, la cotización prevista por el Sistema General de Riesgos Laborales.

3. Un riesgo asegurable, entendido como un hecho cierto y repentino que ocasione un daño en la vida o la integridad física del trabajador.

4. La obligación condicional del asegurador, consistente en la promesa del asegurador de pagar la indemnización tarifada por el Sistema General de Riesgos Laborales, en caso de realizarse el riesgo asegurado.

$5 \quad$ Véase, al respecto, Corte Constitucional. Sentencia C-336 de 2012; Valdivieso y Lombana (2014, pp. 11-16); y Diazgranados (2012-2013).

6 Véase Código de Comercio, artículo 1045, Elementos esenciales del seguro; y Fasecolda (s.f.). 
En este orden de ideas, que el sistema general de riesgos laborales encuentre su fundamento pleno en un modelo de seguro significa que el empleador traslada a un tercero el riesgo de afectación a la salud de sus trabajadores, por causa o con ocasión del desempeño de actividades laborales. Este riesgo creado por el empleador es asumido por una compañía de seguros, que es la Administradora de Riesgos Laborales, cuya función esencial es la de administrar este riesgo a cambio de una prima o cotización, que varía de conformidad con el nivel de exposición al riesgo y la actividad laboral. Se trata pues de un modelo puro de aseguramiento, por cuanto este tipo de compañías están habilitadas de manera específica para desempeñarse en este ramo especial, todo bajo estrictos protocolos legales, contables y financieros que obligan y garantizan una adecuada acumulación de reservas que permitan responder por los siniestros acaecidos.

Como se ha señalado, los componentes del sistema integral de seguridad social son los subsistemas de pensiones, salud, riesgos laborales y servicios sociales complementarios, que están definidos en el artículo 48 de la Constitución Política, la Ley 100 de 1993 y, específicamente para el caso de riesgos laborales, el Decreto 1295 de 1994 y las Leyes 776 de 2002 y 1562 de 2012.

E1 Decreto Ley 1295 de 1994, dispuso que el Sistema General de Riesgos Profesionales obedece al "conjunto de entidades públicas y privadas, normas y procedimientos, destinados a prevenir, proteger y atender a los trabajadores de los efectos de las enfermedades y los accidentes que puedan ocurrirles con ocasión o como consecuencia del trabajo que desarrollan" (art. 1). Esta finalidad se concreta en el establecimiento de objetivos específicos del sistema, que están previstos por el artículo 2 así:

Establecer las actividades de promoción y de prevención tendientes a mejorar las condiciones de trabajo y salud de la población trabajadora, protegiéndola contra los riesgos derivados de la organización del trabajo que puedan afectar la salud individual o colectiva en los lugares de trabajo tales como los físicos, químicos, biológicos, ergonómicos, psicosociales, de saneamiento y de seguridad; // Fijar las prestaciones de atención de la salud de los trabajadores y las prestaciones económicas por incapacidad temporal a que haya lugar frente a las contingencias de 
accidente de trabajo y enfermedad profesional; // Reconocer y pagar a los afiliados las prestaciones económicas por incapacidad permanente parcial o invalidez, que se deriven de las contingencias de accidente de trabajo o enfermedad profesional y muerte de origen profesional, y // Fortalecer las actividades tendientes a establecer el origen de los accidentes de trabajo y las enfermedades profesionales y el control de los agentes de riesgo profesional.

En este sentido, el artículo 8 de esta norma dispone el régimen de transferencia de riesgos laborales, de manera que el accidente producido como consecuencia directa del trabajo o labor desempeñada y la enfermedad que haya sido catalogada como laboral deberán ser asumidos por la ARL.

Como precedente constitucional, la demanda de inconstitucionalidad contra el artículo 216 (parcial) del Código Sustantivo del Trabajo, expresada en Sentencia C-336 de 2012 de la Corte Constitucional, ha señalado que:

“... con la creación de esta forma de aseguramiento, el legislador, con el propósito de proteger a los trabajadores de las contingencias o daños que sufran como consecuencia de la relación laboral, ha impuesto la obligación a los empleadores de trasladar los riesgos a entidades especializadas en su administración, mediando una cotización a cargo exclusivamente del empleador [arts. 16 y 21 del decreto 1295 de 1994] y ha determinado claramente las prestaciones a las que tendrán derecho los trabajadores que se vean afectados por una contingencia de origen profesional [Arts. 5, 6 y 7 del decreto 1295 de 1994]".

La consecuencia de definir este esquema de funcionamiento para la protección del riesgo laboral es que ello implica:

“... que las entidades aseguradoras asumen el riesgo objetivo que en principio tendrían los empleadores pero que por virtud de la ley y en aras de otorgar una mayor protección a los trabajadores, se les traslada a ellas, mediando, una cotización que se encuentra a cargo del patrono. La función que cumplen es entonces preve- 
nir, atender y proteger a la población trabajadora de los efectos causados por accidentes y enfermedades que les puedan ocurrir con ocasión o como consecuencia del trabajo que desarrollan" 7

En este precedente judicial, la Corte Constitucional se refirió de manera expresa a la teoría del riesgo creado, adoptada por nuestro modelo de protección de riesgos laborales, en el siguiente sentido:

"Ahora bien, como lo recuerda el representante del Ministerio de Hacienda y Crédito Público el Sistema de Riesgos Profesionales se estructura a partir de la existencia de un riesgo creado por el empleador. El Legislador acoge en esta materia la teoría del riesgo creado en la que no se toma en cuenta la culpa del empleador, sino que se establece una responsabilidad objetiva por cuya virtud resulta obligado a reparar los perjuicios que sufre el trabajador al desarrollar su labor en actividades de las que el empresario obtiene un beneficio"

Actualmente la Ley, con el propósito de proteger a los trabajadores de las contingencias o daños que sufran como consecuencia de la relación laboral, ha impuesto la obligación a los empleadores de trasladar ese riesgo a entidades especializadas en su administración, mediando una cotización a cargo exclusivamente del empleador [Decreto Ley 1295 de 1994, arts. 16 y 21] y ha determinado claramente las prestaciones a las que tendrán derecho los trabajadores que se vean afectados por una contingencia de origen profesional [Decreto Ley 1295 de 1994, arts. 5, 6 y 7$]$.

En ese orden de ideas, las entidades Administradoras de Riesgos Profesionales, bajo un esquema de aseguramiento, -en el que las coti-

$7 \quad$ Corte Constitucional, Unidada Normativa. Sentencia C-453 de 12 de junio de 2002. Acción pública de inconstitucionalidad contra el artículo 9 parcial del Decreto Ley 1295 de 1994, "por el cual se determina la organización y administración del sistema general de riesgos profesionales". M. P. Álvaro Tafur Galvis; y Corte Constitucional. Sentencia C-250 de 16 de marzo de 2004. Demanda de inconstitucionalidad contra el artículo 16 parcial del Decreto Ley 1295 de 1994, "por el cual se determina la organización y administración del Sistema General de Riesgos Profesionales"; reiteradas en la referida Sentencia C-336 de 2012.

8 En relación con la evolución de la legislación en este campo y la consagración de la teoría del riesgo creado, véase Corte Suprema de Justicia. Sala de Casación Laboral, Sección Segunda, Sentencia del 13 de julio de 1993, Acta 37. M.P. Hugo Suescún Pujols. 
zaciones o primas que el empleador entrega al sistema por cada uno de los trabajadores afiliados generan una mutualidad o fondo común, con el cual se financian las prestaciones anotadas-, deben ocuparse de brindar a los trabajadores la prestación de los servicios de salud que requieran, así como asumir el reconocimiento y pago oportuno de las prestaciones económicas establecidas en el Decreto Ley 1295 de 1994 -incapacidad temporal, incapacidad permanente parcial, pensión de invalidez, pensión de sobrevivientes, auxilio funerario-, al tiempo que deben realizar actividades de prevención, asesoría y evaluación de riesgos profesionales, y promover y divulgar programas de medicina laboral, higiene industrial, salud ocupacional y seguridad industrial [Decreto Ley 1295 de 1994, art. 80].

De la misma manera, puntualizó que este es el mismo criterio asumido por la Corte Suprema de Justicia, Sala de Casación Laboral, señalando que el artículo 216 del Código Sustantivo del Trabajo ${ }^{9}$ se constituye en un régimen de responsabilidad objetiva distinto al fijado en la creación del Sistema de Riesgos Laborales:

"Respecto a la diferencia de la regulación de estas dos clases de responsabilidades, esto es, la prevista para el Sistema de Seguridad Social Integral - Sistema de Riesgos Profesionales, y la señalada para el empleador que incurra en culpa patronal, en casación del 30 de junio de 2005 radicado 22656, reiterada en decisión del 29 de agosto de igual año radicación 23202, esta Corporación puntualizó: “(...) es del caso precisar que para que se cause la indemnización ordinaria y plena de perjuicios prevista en el artículo 216 del Código Sustantivo del Trabajo exige la ley, amén, obviamente, de la ocurrencia del riesgo, esto es, el accidente de trabajo o la enfermedad profesional, la culpa suficientemente comprobada" del empleador; a diferencia de lo que ocurre con las prestaciones económicas y asistenciales tarifadas previstas, hoy, en los artículos 249 y siguientes de la Ley 100 de 1993, Ley 776 de 2002 y demás normas que las reglamentan,

9 Código Sustantivo del Trabajo: "Artículo 216. Culpa del empleador. Cuando exista culpa suficiente comprobada del empleador en la ocurrencia del accidente de trabajo o de la enfermedad profesional, está obligado a la indemnización total y ordinaria por perjuicios, pero del monto de ella debe descontarse el valor de las prestaciones en dinero pagadas en razón de las normas consagradas en este Capítulo". 
especialmente las contenidas en el Decreto 1295 de 1994, que se causan por el mero acaecimiento de cualquiera de las contingencias anotadas, sin que para su concurso se requiera de una determinada conducta del empleador. Dicha diferencia estriba, entonces, esencialmente, en que la segunda de las responsabilidades señaladas, es decir, la del Sistema General de Riesgos Profesionales, es de carácter eminentemente objetivo, de modo que, para su definición, basta al beneficiario de las prestaciones que de ella se desprende acreditar el vínculo laboral y la realización del riesgo con ocasión o como consecuencia del trabajo; en tanto que, la responsabilidad que conlleva la indemnización ordinaria y total de perjuicios tiene una naturaleza subjetiva, de modo que, su establecimiento amerita, además de la demostración del daño a la integridad o a la salud del trabajador con ocasión o como consecuencia del trabajo, la prueba del incumplimiento del empleador a los deberes de protección y seguridad que, según lo señalado por el artículo 56 del Código Sustantivo del Trabajo, de modo general le corresponden.

Este sistema dual de responsabilidad asegura, por una parte, que el Sistema General de Riesgos Profesionales cubra los riesgos que por su propia naturaleza genera el trabajo; y, de otro lado, que los daños ocasionados al trabajador por conducta culposa y dolosa de su empleador le sean resarcidos total y plenamente, atendiéndose el régimen general de las obligaciones. // Dichas responsabilidades comportan un nexo de causalidad entre el trabajo y el accidente de trabajo o la enfermedad profesional que afectan la salud o integridad del trabajador. Nexo que, en términos del accidente de trabajo, se produce "por causa o con ocasión del trabajo”, como lo prevé el artículo 9 del Decreto 1295 de 1994; y, en materia de enfermedad profesional, como consecuencia obligada y directa de la clase de trabajo que desempeña el trabajador, como lo dice el artículo 11 ibidem" $\underline{10}$.

El esquema legal y judicial del Sistema General de Riesgos Laborales implica que la naturaleza del Sistema de Riesgos Laborales se concentra en la

10 Corte Suprema de Justicia, Sala Laboral. Sentencia del 1 de junio de 2010, donde se reitera lo dicho por esta Corporación en sentencia radicación 27501 del 4 de julio de 2006. 
disposición de recursos para asumir garantías esenciales para los trabajadores. En este sentido, "la lógica que subyace a esta dinámica implica que el diseño de este sistema debe privilegiar la liquidez de quien acopia el dinero, pues su capacidad para responder por las garantías en cuestión depende ello" 11 . Es clara la Corte en manifestar que surgen dos consecuencias de este esquema:

- Es necesario que el manejo de los recursos para este fin, por parte de quienes los administran, se oriente a reunir reservas presupuestales proporcionales a las eventuales obligaciones.

- La garantía respaldada en el adecuado manejo de estos recursos lleva aparejada la disponibilidad permanente e incondicional de los activos, pues se trata de obligaciones derivadas de la imposición normativa (constitucional y legal) de proteger a los trabajadores. De ahí que la responsabilidad que la sostiene sea objetiva.

\section{LA OBLIGACIÓN GENERAL DE PROTECCIÓN Y SEGURIDAD PARA CON LOS TRABAJADORES}

Se trata de una obligación que se encuentra en múltiples disposiciones contenidas en el Código Sustantivo del Trabajo - CST y del Sistema General de Riesgos Laborales - SGRL, en los cuales están incluidas las de prevención de accidentes y enfermedades laborales, y el mejoramiento de las condiciones de trabajo ${ }^{12}$.

En este sentido, las disposiciones sustantivas laborales de Seguridad y Salud en el Trabajo, así como las del Sistema General de Riesgos

11 Corte Constitucional. Sentencia C-460 de 17 de julio de 2013. Demanda de inconstitucionalidad contra el artículo 1 parcial de la Ley 1539 de 2012, "por la cual se implementa el certificado de aptitud psicofísica para el porte y tenencia de armas de fuego y se dictan otras disposiciones", Énfasis agregado.

12 Los artículos 57 num. 2 y 348 del CST, establecen la obligación a cargo de los empleadores, de suministrar y acondicionar locales y equipos de trabajo adecuados, elaborar y ejecutar programas de seguridad y salud en el trabajo, para proteger a los trabajadores de accidentes de esa índole y enfermedades laborales. También se encuentra el art. 2 de la Resolución 2400 de 1979 y lit. a) del 84 de la Ley 9 de 1979, que ordenan al empleador proveer y mantener un ambiente de trabajo en adecuadas condiciones de higiene y seguridad. 
Laborales, han sido unívocas en comprometer al empleador a cuidar y procurar por la seguridad y salud de los trabajadores, y adoptar todas las medidas a su alcance, con el fin de prevenir los accidentes y enfermedades profesionales puesto que "la salud de los trabajadores es una condición indispensable para el desarrollo socio-económico del país, su preservación y conservación son actividades de interés social y sanitario" $\underline{13}$.

\section{LAS REGLAS DE LA CORTE SUPREMA DE JUSTICIA PARA DETERMINAR LA RESPONSABILIDAD PLENA DEL EMPLEADOR. PRECEDENTE JUDICIAL 2019}

Estas son las reglas más relevantes, según la Corte Suprema de Justicia en el año 2019, para determinar la responsabilidad plena de perjuicios a cargo del empleador:

i. Imprevisión del empleador y el incumplimiento de sus deberes de protección y seguridad en la salud del trabajador ${ }^{14}$, esto significa que no es suficiente con cumplir con un programa de salud en el trabajo (sic) $\frac{15}{}$, sino también por no tomar las precauciones necesarias para la ejecución de la tarea encomendada. Así pues, la indemnización se genera cuando quien tiene el deber de seguridad no lo acata y no despliega una acción protectora, la cual se concreta en la adopción de todas las medidas necesarias para que el empleado no sufra lesión alguna durante el ejercicio de la tarea, o en su defecto, disminuye los riesgos asociados a ella.

ii. El empleador responde por el daño causado por sus trabajadores, en los términos del artículo 32 del CST. En consecuencia, a pesar de la existencia del empleador, las personas que lo representan tienen a su vez el poder de subordinación sobre otros y

13 C.S.J. SL9355-2017, reiterada en la sentencia CSJ SL12862-2017. Ver Artículo 81 de la Ley 9 de 1979.

14 C.S.J SL1361-2019. Radicación n. ${ }^{\circ}$ 61560. Bogotá, D.C., trece (13) de marzo de dos mil diecinueve (2019). Ver también C.S.J. SL786-2019. Radicación n. ${ }^{\circ}$ 59843. Bogotá, D.C., trece (13) de marzo de dos mil diecinueve (2019).

15 En la actualidad se denomina sistema de gestión de seguridad y salud en el trabajo. 
pueden en un momento dado comprometer a la empresa mediante sus actos u omisiones culposos inherentes a su función $\frac{16}{}$.

iii. Omisión de implementar seguridad y salud en el trabajo. La responsabilidad plena se encuentra en la omisión del empleador de sus obligaciones de identificar y comunicar al trabajador la exposición que la actividad laboral puede generar en su salud y adoptar todas las medidas tendientes a evitar una afectación en su salud ${ }^{17}$.

iv. La concurrencia de culpas no exonera al empleador ${ }^{18}$ : Dos son las reglas importantes en este punto:

a. Si el cumplimiento de todas las normas de seguridad se produce y el siniestro acaece, actúan como eximentes de responsabilidad del empleador la existencia de una fuerza mayor o de un caso fortuito, la culpa exclusiva de la víctima o el hecho de un tercero ${ }^{\frac{19}{}}$.

b. Sin embargo, no le liberan de responsabilidad, en el caso del incumplimiento de los mandatos de seguridad, la concurrencia de culpa con el trabajador accidentado, porque la responsabilidad de la empresa en el accidente laboral no admite compensación de culpas, conforme al artículo 216 del $\mathrm{CST}^{20} \underline{\text { }}$.

v. Exoneración por culpa suficientemente comprobada del empleado, pues la actuación que desarrolló el trabajador fallecido y que generó el accidente de trabajo, no solo se dio sin la aquiescencia de la sociedad empleadora, sino también sin su conocimiento y al margen de su poder de subordinación funcional. $\underline{21}$

16 C.S.J. SL651-2019. Radicación n. ${ }^{\circ 66367 . ~ B o g o t a ́, ~ D . ~ C ., ~ c i n c o ~(5) ~ d e ~ m a r z o ~ d e ~ d o s ~ m i l ~}$ diecinueve (2019).

17 C.S.J. SL1492-2019. Radicación n. ${ }^{\circ}$ 69107. Bogotá, D. C., dos (2) de abril de dos mil diecinueve (2019).

18 C.S.J. SL904-2019. Radicación n. ${ }^{\circ}$ 59965. Bogotá, D. C., diecinueve (19) de marzo de dos mil diecinueve (2019).

19 Ver sentencias CSJ SL12862-2017 y CSJ SL14420-2014, reiterada en sentencia CSJ SL15114-2017.

20 Ver CSJ SL5463-2015.

21 C.S.J SL1535-2019. Radicación n. ${ }^{\circ}$ 70325. Bogotá, D. C., treinta (30) de abril de dos mil diecinueve (2019). Ver también C.S.J. SL1337-2019. Radicación n. ${ }^{\circ}$ 60926. Bogotá, D. C., diecinueve (19) de febrero de dos mil diecinueve (2019). 


\section{CONCLUSIONES}

i. La indemnización plena de perjuicios por la que responde el empleador depende de que se demuestre en juicio los siguientes supuestos: i) la ocurrencia del accidente de trabajo o la enfermedad profesional; ii) los perjuicios padecidos a consecuencia de estos; iii) la culpa del empleador y iv) la relación de causalidad entre el hecho y el perjuicio.

ii. El empleador responde por la culpa leve, en los términos del artículo 63 del Código Civil, razón por la cual, es necesario determinar la causa del suceso.

iii. Las reglas determinadas por la Corte Suprema de Justicia señalan un camino inequívoco: la responsabilidad derivada del cuidado de la salud del trabajador requiere de una labor coordinada y estrecha entre empleador, ARL y trabajador.

iv. La separación entre la responsabilidad objetiva y subjetiva derivada del riesgo laboral es cada vez más estrecha, razón por la cual, cobra mayor importancia ampliar y profundizar la cultura de la prevención, así como la implementación de los sistemas de seguridad y salud en el trabajo.

\section{BIBLIOGRAFÍA}

Arenas Monsalve, G. (2011). El derecho colombiano de la seguridad social. Bogotá: Legis.

Avella, M. (2012). Las instituciones laborales en Colombia. Contexto histórico de sus antecedentes y principales desarrollos hasta 1990. Bogotá: Universidad Jorge Tadeo Lozano. 
Cañón, L. (2017a). La solidaridad como fundamento del Estado social de derecho, de la seguridad social y la protección social en Colombia. Páginas de Seguridad Social, 1(1), 5-29.

Cañón Ortegón, L. (2017b). Una visión integral de la seguridad social. Bogotá: Universidad Externado de Colombia.

Conte-Grand, A. H. y Rodríguez, C. A. (1999). Cobertura de los riesgos del trabajo. Manual con experiencias actuales y alternativas. Santiago de Chile: OIT.

Cortés Gonzalez, J. C. (2015). Régimen de los riesgos laborales y de la seguridad y salud en el trabajo. Bogotá: Legis.

Cortés Reyes, E. (ed.). (2015). Sistema General de Riesgos Laborales en Colombia. Suficiencia y variación de la tasa de cotización. Bogotá: Universidad Nacional.

Diazgranados Quimbaya, L. A. (2012-2013). Régimen de los Riesgos Laborales en Colombia Aproximación a la reforma 2012. Liberty Seguros ARL.

Econometría S.A. (2018). Estudio sobre beneficios sociales y económicos del sistema general de riesgos laborales. Ciudad: Editor.

Fasecolda (s.f./a). Aspectos básicos del contrato ABC) de seguros. Fasecolda.com. Recuperado de https://fasecol da.com/files/7 013/8626/3 513/jul-aspectos_bsicos_del_contrato_abc_de_s eguros.pdf

Gómez C., C. E. (2015). Principales hitos de la evolución normativa de los riesgos laborales en Colombia. En Historia avances y proyección de la Seguridad Social en Colombia (pp. 186-199). Bogotá: Organización Iberoamericana de la Seguridad Social - OISS.

ICSS - Instituto Colombiano de Seguro Social (1967). Acuerdo 241. Reglamento de Prevención de Riesgos Profesionales.

ISOTools Excellence (2015). Riesgo laboral, ¿cuál es su definición? 15 de julio. Recuperado de https://ww w.isotool s.cl/riesgo -laboral-d efinicion/ 
Lang, F. (1947). Workmen's Compensation Insurance. Monopoly or Free Competition? Chicago: Richard D. Irwin.

Larenz, K. (1958). Derecho de las obligaciones. Revista de Derecho Público, 2.

Plazas, G. (2017). La nueva práctica laboral 2017-2018. Bogotá: Consejo Colombiano de Seguridad.

Puyana, A. (2013). El sistema general de riesgos laborales en Colombia. Bogotá: Univeridad Externado de Colombia.

Rodríguez, C. A. (2009). Los convenios de la OIT sobre seguridad y salud en el trabajo: una oportunidad para mejorar las condiciones y el medio ambinete de trabajo. Buenos Aires: OIT en Argentina.

Rodríguez, R. (2016). Sistema General de Riesgos Laborales. Bogotá: Universidad del Norte y Grupo Editorial Ibáñez.

Sánchez Acero, D. A. (2015). Un nuevo concepto de culpa patronal. Bogotá: Universidad Externado de Colombia.

Valdivieso Rueda, D. F. y Lombana Sierra, J. I. (2014). Accidente o enfermedad laboral por culpa del empleador. Revista Actualidad Laboral, 184, 11-16.

Varela Collazos, G. (2016). El sistema general de riesgos laborales. Bogotá: Consejo Superior de la Judicatura.

Velásquez, L. F. (2016). Compendio comentado de normas legales en Colombia sobre seguridad y salud en el trabajo. Bogotá: Mundo del Libro. 